\begin{tabular}{|c|c|c|c|c|}
\hline $\begin{array}{c}\text { REVISTA ENTORNO } \\
\text { GEOGRÁFICO }\end{array}$ & $\begin{array}{c}\text { Julio/ } \\
\text { Diciembre } \\
2019\end{array}$ & $\mathrm{~N}^{\circ} 18$ & pp. 148-171 & $\begin{array}{c}\text { ISSN (en línea): 2382-3518/ ISSN } \\
\text { impreso: 1692-0074 }\end{array}$ \\
\hline
\end{tabular}

DOI: $10.25100 /$ eg.v0i18.8893

(c) $(1) \Theta \Theta$

Espacios y Territorios

\title{
Acceso a la tierra y su relación con la autonomía alimentaria en el corregimiento de Bitaco municipio de La Cumbre Valle del Cauca, Colombia
}

\section{The relationship between land acces and food autonomy in bitaco village, municipality of La Cumbre Valle del Cauca, Colombia}

\section{Lorena Castañeda Zuluaga}

Geógrafa, Departamento de Geografía, Universidad del Valle. Santiago de Cali, Colombia.

ORCID: 0000-0002-6977-179.

Correo electrónico: I.lorenacz@gmail.com

Para citar este artículo: Castañeda Zuluaga, L. (2019). Acceso a la tierra y su relación con la autonomía alimentaria en el corregimiento de Bitaco municipio de La Cumbre Valle del Cauca, Colombia. Entorno Geográfico, (18). DOI: 10.25100/eg.v0i18.8893.

Resumen: La concentración de la propiedad de la tierra ha afectado en gran medida a la producción agrícola de nuestro país, tanto que las dinámicas actuales del capital económico amenazan seriamente el derecho a una alimentación adecuada. En el caso de Colombia, observamos una situación problemática en donde las comunidades campesinas están siendo despojadas de sus territorios, para ser entregados a monopolios y empresas trasnacionales. la concentración de la tierra por parte de estas empresas ocasiona múltiples contrariedades entre ellas; una notoria reducción de la tierra y extracción de los recursos naturales lo cual, impide desarrollar las diferentes actividades agrícolas productivas de las comunidades campesinas.

Palabras clave: Autonomía alimentaria, campesinos, agricultura.

Abstract: The concentration of land ownership has greatly affected the agricultural production of our country, so much so that the current dynamics of economic capital seriously threaten the right to adequate food. In the Colombian case, we observe a problematic situation where peasant communities are being stripped of their territories, to be handed over to monopolies and transnational 
corporations. The concentration of land by these companies causes multiple contradictions between them; a noticeable reduction of the land and extraction of natural resources which prevents the

Recibido: 2 de julio de 2019

\section{INTRODUCCIÓN}

L. cuestión agraria en Colombia ha estado marcada históricamente por el problema de acceso a la propiedad por parte de pequeños y medianos productores, o por quienes no disponen de este activo productivo. También ha sido notoria la debilidad de los derechos de propiedad y la incapacidad del Estado para garantizarlos. La lucha por acceder a la tierra en el sector rural ha dado lugar a innumerables conflictos, entre los campesinos, el Estado, la sociedad rural y la urbana (Absalón, 2009). El problema agrario configura una serie de conflictos en cada una de las facetas, es decir que existen varios puntos focales sobre los problemas de la cuestión agraria, entre ellos: Conflictos económicos, sociales, políticos institucionales y ambientales, cada uno de estos conflictos evidencia las problemáticas que enfrenta Colombia actualmente (Machado, 1998).

Por otro lado, la aparición de nuevas políticas agrarias en Colombia, ha afectado en gran medida el desarrollo rural, pues a finales de la década de los ochenta e inicios de los noventa Colombia presento cambios significativos en cuanto a la estructura productiva development of the different productive agricultural activities of the rural communities.

Keywords: Food autonomy, farmers, agricultural.

Aceptado: 14 de enero de 2020

agrícola, los cuales son atribuidos principalmente al proceso de apertura económica, siendo beneficiada la economía capitalista y viéndose en desventaja la economía campesina (Beltrán Gutiérrez y Gutiérrez Rozo, 2007). La Reforma Agraria en Colombia es un tema estratégico para la solución de diversas problemáticas que afectan al país desde hace años, como son la violencia en el campo, el desplazamiento forzado, el desempleo, la pobreza y el narcotráfico (Franco y De los Ríos, 2011).

Para Machado (1998) otro de los conflictos que existe, es la acentuada migración rural-urbana impulsada por la disminución de áreas cultivadas, el difícil acceso de la población rural a la tierra y servicios del Estado, sin que esa población encuentre alternativas de empleo e ingreso por fuera de la agricultura. En ello se conjugan el fenómeno de los desplazados por la guerra y las violencias en el sector rural. El avance del minifundio y la fragmentación antieconómica de la propiedad rural, acompañada de altos índices de pobreza rural; desasosiego social y enfrentamiento de la población rural con el Estado y sus instituciones. Al mismo tiempo, un deterioro de la mediana 
propiedad y de la clase media rural que impide lograr equilibrios en la estructura.

En este listado de conflictos anteriormente señalados, pude decirse que el problema agrario en Colombia no se ha resuelto todavía, siendo esto fundamental para poder lograr un desarrollo sostenible social, político, económico e institucional, pero la solución a este problema agrario no solo es un asunto sectorial, es de toda la estructura social, política y económica del país (Machado, 1998).

De este modo, la importancia en este proyecto de investigación radica, en poder evaluar cuáles han sido las causas principales de los cambios en los procesos productivos campesinos, con el fin de poder evidenciar la relacion que hay entre esos cambios y la autonomia alimentaria de la comunidad del corregimiento de Bitaco.

\section{ÁREA DE TRABAJO}

El corregimiento de Bitaco (Figura 1) se encuentra localizado en el sur occidente de Colombia, en el Departamento del Valle del Cauca Municipio de La Cumbre, con altura de $1.470 \mathrm{msnm}$ y comprende la zona de reserva forestal, del mismo nombre, declarado por la CVC. Posee gran parte de la zona de bosques naturales, agrupa la mayor parte hídrica del Municipio y es atravesado por el río Bitaco (Enríquez, 2004). El corregimiento de Bitaco, cuenta con un área total de 3312 Ha y 2696 habitantes (CVC, 2003).

Fuente: (Corporación Autónoma regional del Valle del Cauca, 2017)

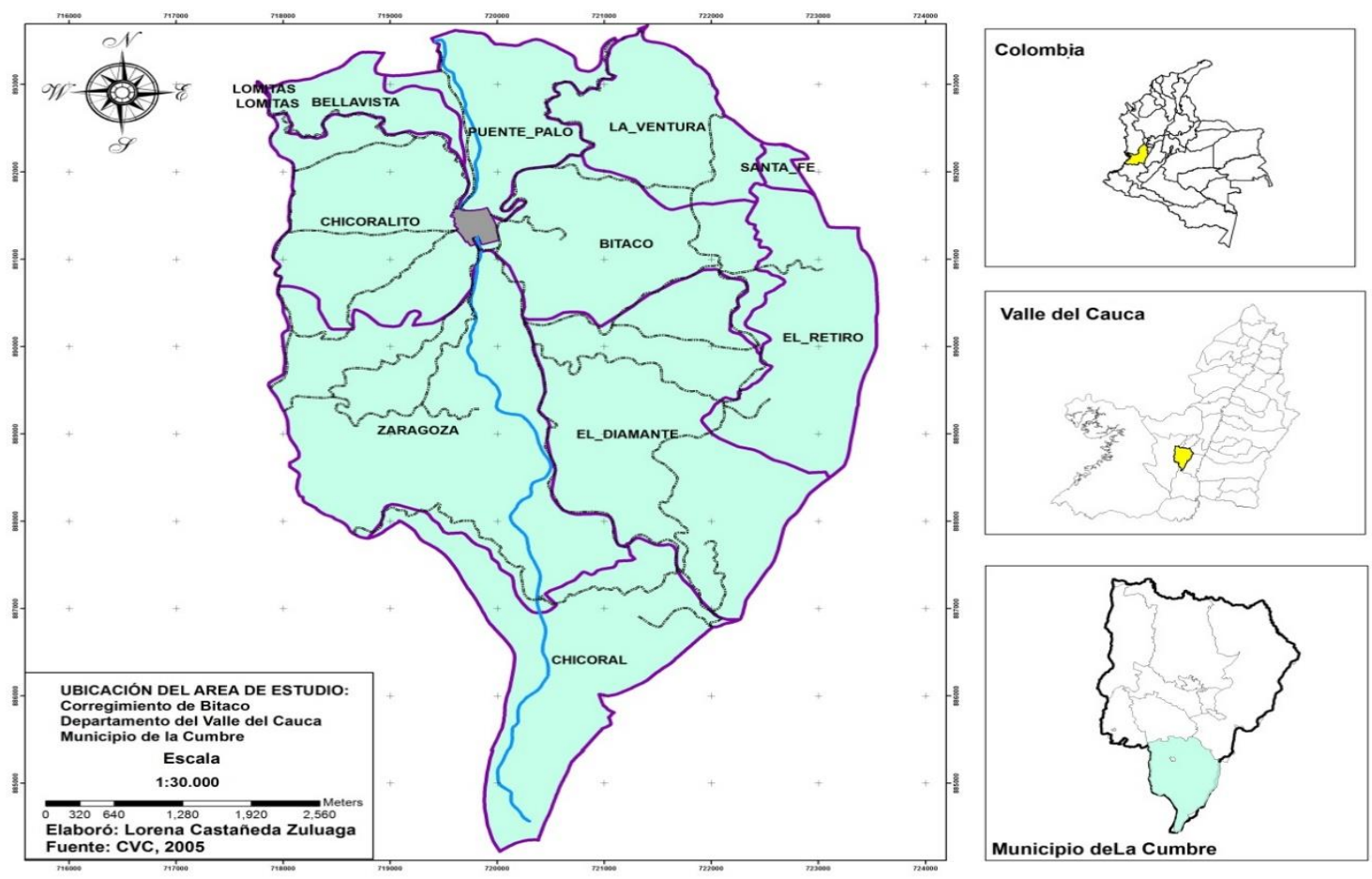

Figura 1. Ubicación del área de estudio. 


\section{SOPORTE TEÓRICO}

Para respaldar la investigación se tomaron algunas recopilaciones teóricas y conceptuales referentes a la soberanía alimentaria, seguridad alimentaria y autonomía alimentaria. Cada una de estas diferentes concepciones teóricas permite evaluar y soportar el desarrollo de la investigación.

\section{Soberanía alimentaria}

El concepto de Soberanía Alimentaria fue expresado por primera vez por el movimiento Vía Campesina en la Cumbre Mundial de la Alimentación realizada en Roma en 1996. De acuerdo a esto, la soberanía alimentaria se define como:

"El derecho de los pueblos, comunidades y países a definir sus propias políticas agrícolas, pastoriles, laborales, de pesca, alimentarias y agrarias que sean ecológica, social, económica y culturalmente apropiadas a sus circunstancias exclusivas. Esto incluye el derecho real a la alimentación y a la producción de alimentos, lo que significa que todos los pueblos tienen el derecho de tener alimentos y recursos para la producción de alimentos seguros, nutritivos y culturalmente apropiados, así como la capacidad de mantenerse a sí mismos y a sus sociedades" (Grain, 2005).

En una dimensión más amplia la soberanía alimentaria es una alternativa sólida a la corriente de pensamiento oficial sobre la producción de alimentos. La lucha por la soberanía alimentaria incorpora temas tan vastos como la reforma agraria, el control territorial, los mercados locales, la biodiversidad, la autonomía, la cooperación, la deuda, la salud y muchos otros temas que tienen una importancia fundamental para poder producir alimentos localmente (Grain, 2005).

La reforma agraria, en especial, es un componente importante de la soberanía alimentaria una redistribución radical de la tierra, particularmente entre los más pobres y quienes no tienen acceso a ella. Uno de los principales cuellos de botella de la producción local de alimentos es la distribución desigual de la tierra. En muchos países del mundo, el 20\% de los terratenientes controlan el $80 \%$ de la tierra y esa tierra a menudo es utilizada para producir productos básicos para exportación, en lugar de alimentos a los que pueda acceder la población local. De manera similar, la aplicación de los derechos de los pueblos indígenas a sus territorios es un requisito indispensable para avanzar hacia la soberanía alimentaria (Grain, 2005).

\section{Seguridad alimentaria}

El concepto de seguridad alimentaria tiene varias interpretaciones. Usando los primeros conceptos que acuño la FAO seguridad alimentaria se define de la siguiente manera. 
"por seguridad alimentaria nacional se entiende el logro de una disponibilidad agregada de alimentos basicos suficientes para satisfacer la demanda interna de estos, considerando no solo la demanda efectiva sino tambien la seguridad nutricional de los factores con ingreso insuficientes para alcanzar los minimos normativos. Definido asi, este concepto abarcaria tanto los problemas de regulacion de los factores que afectan de modo coyuntural a la oferta interna (factores climaticos, de comercio internacional, etc.) como aquellos de tipo estructural que conducen a insuficiencias cronicas (y aveces crecientes) en la producción interna de alimentos basicos" (Torres, 1987).

Por seguridad nutricional se entendera el acceso regular en el tiempo y en el espacio a los elementos necesarios para dar pleno desarrollo a las potencialidades biologicas de todos los individuos de la sociedad, cualquiera sea su estatus socioeconomico y cualquiera su localizacion geografica. Según la FAO, el objetivo final de la seguridad alimentaria consiste en garantizar que todas las personas tengan en todo momento acceso fisico y economico a los alimentos basicos que necesiten. El concepto involucra acciones que afectan toda la estructura economica y social y su busqueda implica una politica alimentaria integral, con redistribucion del ingreso, sea directamente o mediante mecanismos indirectos como la distribucion gratuita o subsidiada de los alimentos en los sectores mas vulnerables (Torres, 1987).

\section{Autonomía Alimentaria}

En buena parte, la construcción de este nuevo concepto debe "aterrizar" el concepto de soberanía alimentaria a nivel de comunidades y familias productoras, en vez de contemplarlo a nivel nacional. Esto implica que los pequeños productores: campesinos, indígenas $\mathrm{y}$ afro-descendientes, no sean dependientes. Por esta razón se propone, el concepto de Autonomía Alimentaria de las familias productoras y de las comunidades organizadas (Perret y Zúñiga Vega, s.f).

La propuesta enfatiza en la Autonomía, la cual es un concepto que ya han acuñado algunas organizaciones sociales en Colombia, que además de su definición occidental centrada en el individuo, significa aquí "el auto-gobierno de las comunidades". En ese sentido, el concepto rompe claramente con la concepción nacional-estatal de la soberanía alimentaria. Pero también enfatiza en un aspecto nuevo: en el control por parte de las comunidades de toda la cadena productiva. Eso implica que las familias productoras $\mathrm{y}$ las comunidades rurales no dependan de insumos externos (que sean fertilizantes, plaguicidas, pero también de las semillas), y controlen en buena parte las cadenas de comercialización y no dependan de empresas capitalistas externas ya sean nacionales o multinacionales, entre otras cosas (Perret y Zúñiga Vega, s.f).

La propuesta de la Autonomía Alimentaria pretende construir un mundo 
rural en el cual cada familia y cada comunidad local consuma en prioridad y mayoría lo que produce, que conserve y recupere sus semillas nativas y locales, que tenga una producción agroecológica, diversificada, integrada, con tecnologías apropiadas técnica y culturalmente, comunidades en las que se dé el trueque, que estas familias y comunidades sean los primeros "transformadores" de sus productos, a través de la micro y pequeña industria, y que sus excedentes sirvan para abastecer los mercados de las ciudades cercanas, favoreciendo la comercialización directa sin intermediarios. Por otro lado, se considera que para fortalecer la Autonomía Alimentaria, hace falta fortalecer las organizaciones campesinas, indígenas, afro-descendientes y rurales en general, para que puedan desarrollar estos procesos en sus comunidades, siguiendo los principios y líneas directrices tanto de la agroecología como de las normas propias de cada una de las comunidades (campesinas, indígenas y afrodescendientes) (Perret y Zúñiga Vega, s.f).

Es en este sentido, se hace la apuesta de la agricultura familiar y comunitaria agroecológica, sostenible, autónoma, cuyo proceso de transformación y comercialización no sea jerárquico ni centralizado, y que mejoren decisivamente y definitivamente la calidad de vida de las comunidades rurales, a la vez que la alimentación de las comunidades urbanas. Esta alternativa es la única alternativa sostenible (Perret y Zúñiga Vega, s.f).

\section{MATERIALES Y MÉTODOS}

Con el objetivo de determinar el estado de la autonomía alimentaria y el acceso a la tierra en el corregimiento de Bitaco Municipio de la Cumbre, se plantearon varias acciones que se llevaron a cabo gracias a la participación de la comunidad. Cabe señalar que, cada una de las actividades planteadas fueron ejecutadas en la cabecera del corregimiento de Bitaco, con la asistencia de varios campesinos.

\section{Actividad de cartografía social a partir de la visión de los agentes sociales}

La actividad de cartografía social es un ejercicio colectivo de reconocimiento del entorno socio territorial, a través de la construcción de mapas; con este ejercicio se evidencian las relaciones sociales, se develan saberes que permiten a sus participaciones un mejor conocimiento de su realidad, se generan espacios de reflexión y permite construir un lenguaje común de interpretación de las diferentes percepciones de la realidad presente en las comunidades (López, 2004).

Con el objetivo de poder obtener la percepción de los habitantes con respecto a su visión presente, pasado y futura del corregimiento, se recurrió a la 
metodología de cartografía social, la cual fue explicada a los participantes previamente. De igual manera se dió una breve introducción al término de cartografía social y una serie de preguntas orientadoras para que el grupo de participantes plasmaran su percepción en cada uno de los mapas planteados (pasado, presente y futuro)

\section{Identificación de los principales} acontecimientos socio-ambiéntales desde la perspectiva de los agentes sociales en el corregimiento de Bitaco

Una vez realizada la actividad de cartografía social se procedió a realizar la actividad de la línea del tiempo. El objetivo de esta actividad se basa principalmente en la identificación de aspectos sociales, políticos, económicos o ambientales que han generado cambios en la comunidad campesina del corregimiento, en este caso no se propone ninguna fecha de inicio, ya que la idea es que los participantes inicien con el evento que marco más importancia en el corregimiento, los integrantes están en total libertad para la realización de esta actividad.

\section{Identificación de cambios en la autonomía alimentaria campesina.}

Con el objetivo de poder establecer como se encuentra la autonomía alimentaria del corregimiento de Bitaco, se procede a realizar una serie de entrevistas, lo cual permite confrontar los resultados arrojados de las actividades anteriormente diseñadas y desarrolladas en la investigación, de este modo, esta actividad se convierte en un insumo importante para establecer y diferenciar los cambios más representativos que han existido en el corregimiento en cuanto a las diferentes actividades agrícolas realizadas por los campesinos del corregimiento. Así mismo, permite evaluar como los aspectos sociopolíticos han generado de alguna manera impactos negativos y cambios en las producciones agrícolas de la zona.

\section{Entrevistas estructuradas y semi- estructuradas}

El objetivo prioritario de la entrevista semi-estructurada es proporcionar un marco dentro del cual los entrevistados puedan expresar su propio modo de sentir con sus propias palabras; acceder a la perspectiva del sujeto estudiado, comprender sus categorías mentales, sus interpretaciones, sus percepciones sus sentimientos y los motivos de sus actos, teniendo un orden preestablecido en el desarrollo de las preguntas (Idárraga Bahos, 2012). No obstante, el objetivo fue diseñar preguntas abiertas creando la posibilidad de recibir más matices de la respuesta, para poder ir entrelazando temas.

En este sentido, las entrevistas estructuradas previamente se planifican con un determinado orden y contiene un conjunto de categorías u opciones para que el entrevistado elija, Se aplica en forma rígida a todos los sujetos del estudio. Tiene la ventaja de la sistematización, la cual facilita la 
clasificación y análisis, asimismo, presenta una alta objetividad y confiabilidad (Díaz Bravo, Torruco García, Martínez Hernández, \& Varela Ruiz, 2013).

De esta manera, para el desarrollo de la presente actividad, se realizó una serie de entrevistas a diferentes actores de la comunidad del corregimiento de Bitaco, con el objetivo de poder recoger la percepción que tiene cada uno de los actores encuestados sobre su territorio y como este ha cambiado a través del tiempo. Cabe aclarar que para el desarrollo de las entrevistas se realizó simultáneamente un recorrido de campo por la zona de estudio iniciando en la cabecera del corregimiento de Bitaco y culminando en la vereda la Ventura.

Se realizó una actividad de observación del territorio con el objetivo de examinar el uso económico y las diferentes actividades productivas que tienen los predios en el corregimiento. Durante el recorrido por la zona de estudio, fue fácil generar un espacio de empatía y confianza con las personas participes de la actividad lo cual, facilito de alguna manera la realización de las entrevistas diseñadas. Es pertinente mencionar que las entrevistas realizadas fueron sintetizadas en forma de narración para evitar que este se convierta en un análisis monótono y tedioso.

Finalmente, con el desarrollo de esta actividad permitió identificar cuáles son las prácticas agrícolas que se realizan actualmente en el corregimiento, como era la actividad agrícola hace más de 20 años, porque se han dado algunos cambios en cuanto a su estructura agrícola y evidenciar como se encuentra el uso de algunos predios de la zona. Esta actividad se convirtió en un insumo importante y complementario para el desarrollo del segundo objetivo de investigación.

\section{RESULTADOS Y DISCUSIÓN}

En las zonas rurales de Colombia es cada vez más difícil alcanzar un nivel de vida digno; el mensaje que la sociedad colombiana ha enviado a los pobladores rurales ha sido que su progreso o el de sus familias dependen de abandonar el campo. Por esa vía, las ciudades pujantes y modernizadoras dieron la espalda a la sociedad rural dando pie a que muchos de sus conflictos se hubieran agudizado o no hubieran encontrado canales institucionales para ser tramitados. Esto la ha llevado a una especie de crisis permanente y a una condición de inestabilidad (Programa de las Naciones Unidas para el desarrollo, 2012).

Según el censo de 2005 el Municipio de la Cumbre se dedica a actividades laborales relacionadas básicamente con el comercio, la construcción e industria, que en conjunto generan ocupación para el $37 \%$ de la población de la cabecera, contra un $15.7 \%$ que genera la actividad agrícola. El ingreso per cápita de las familias del municipio es muy bajo, generado en parte por las pocas 
oportunidades de empleo y el carácter informal de las actividades laborales de muchos de sus pobladores, cuyos ingresos en algunos casos no alcanzan un salario mínimo mensual (Alcaldía de la Cumbre Valle del Cauca, 2014).

En el caso del corregimiento de Bitaco, los ingresos económicos provienen de diferentes actividades; La principal fuente de empleo en la zona es el cultivo de té el cual, genera 140 empleos directos en $\mathrm{La}$ Cumbre, otra de las fuentes de empleo que existe en el corregimiento provienen especialmente del cultivo de flores. Hacia la parte baja del corregimiento la producción de café genera algunos ingresos, así como los cultivos de pino pertenecientes a la empresa multinacional Smurffit Kappa Cartón de Colombia (Alcaldía de la Cumbre Valle del Cauca, 2014). Las estadísticas del Censo de 2005, muestran que el $60 \%$ de la población del municipio de la Cumbre se dedica a actividades agrícola y de ganadería, el segundo ítem de la generación de empleo se da en la construcción, de los cuales el $7 \%$ de la población realiza estas actividades. El comercio y actividades que tienen que ver con la industria son las ramas que en orden de importancia soportan el empleo en el municipio (CVC, 2006).

Para el 2006 el porcentaje de ingresos económicos en el corregimiento de Bitaco se distribuye de la siguiente manera: En la vereda el Chicoral el $47 \%$ de la población devenga un salario mínimo, el $16 \%$ cuenta un ingreso de más de un salario mínimo, el $16 \%$ obtiene menos de un salario mínimo y el 5\% devengan medio salario mínimo. Una buena parte de los predios son casas de veraneo, lo cual muestra la dinámica económica y productiva de la zona (CVC, 2006).

En la vereda de Chicoral el $25 \%$ de los habitantes deriva sus ingresos de su trabajo como mayordomos en las fincas, el $30 \%$ son amas de casa, el $20 \%$ se encuentran desempleados con ocupación en actividades ocasionales como, construcción, carpintería, agricultura o artesanía, el $20 \%$ son estudiantes y el restante $15 \%$ trabajan en la recolección del té en la hacienda Agrícola Himalaya. Las características de las veredas de Chicoral y Zaragoza es la de propietarios ausentistas, pues predominan las fincas de recreo. Dadas las condiciones biofísicas de la vereda de Chicoral y su vocación forestal como zona de protección, el $55.3 \%$ de los predios no presenta ningún tipo de producción, el $44 \%$ restante se dedica a la producción de heliconias y anturios y un $10,5 \%$ se dedica a la producción de pancoger, Existe también actividades dedicadas a la ganadería extensiva.

\section{Acceso a la tierra en el corregimiento de Bitaco}

En este sentido, existe un factor que perturba en gran medida la producción de alimentos y evita tener un buen desarrollo agrícola en la zona y es que el corregimiento de Bitaco, presenta una alta concentración de la propiedad, el índice de Gini se encuentra en un 66 para el año 
2005, evidenciando que la mayor parte de los predios se encuentra en mano de pocos propietarios. La mayoría de los predios en el corregimiento de Bitaco son fincas con destino para recreación y descanso, de igual forma, existe un alto componente de inmigración de personas procedentes de la ciudad de Santiago de Cali hacia el corregimiento, en especial los fines de semana, para realizar actividades de turismo $\mathrm{y}$ descanso personas (Alcaldía de la Cumbre Valle del Cauca, 2014).

Hace más de 20 años, los campesinos eran dueños de grandes extensiones de tierra, pero decidieron vender su finca por lotes de 200 o 300 metros, este tipo de actividad se hizo por necesidad económica o en la mayoría de los casos la población vieja moría dejando las tierras en herencia a sus hijos y por ende vendían la finca por lotes. Actualmente, los campesinos que no cuentan con suficiente tierra para cultivar deciden ir a alquilar tierras a propietarios con mayor extensión de área para realizar diferentes actividades de agricultura o de ganadería. Es así, como la fragmentación de la propiedad de la tierra en Bitaco se ha dado a diferentes factores tanto internos como externos ${ }^{1}$.

Por lo tanto, para que exista una autonomía alimentaria debe existir al menos una distribución equitativa de la

\footnotetext{
${ }^{1}$ Información recogida en campo a través de entrevistas a los habitantes del corregimiento de Bitaco.
}

tierra, con el objetivo de que la comunidad campesina tenga la capacidad de producir sus propios alimentos y no depender de otros actores para poder suplir su alimentación. Durante el desarrollo de diferentes actividades de cartografía social, entrevistas, análisis de datos catastrales y recorridos por el corregimiento de Bitaco, se evidencio que este, tiene una gran variedad de producción de alimentos, sus condiciones climáticas y las propiedades del suelo hacen que se convierta en una zona con buenas condiciones para la producción agrícola.

Claramente, el Corregimiento de Bitaco produce gran variedad de alimentos entre ellos; (lechugas, tomates, café, platano, sabila, nopal, frijoles), pero el acceso a estos alimentos es limitado $^{2}$ ya que no están disponibles para los habitantes de la zona y estos son enviados para comercializarlos a otras ciudades. En el corregimiento solo quedan los productos de menor calidad.

La autonomía alimentaria, se convierte en un paso necesario para alcanzar la soberanía alimentaria, en este sentido el concepto de autonomía alimentaria es un hecho de autodeterminación, resistencia y lucha por la permanencia cultural de las comunidades. Las estrategias nacionales de soberanía y las políticas de seguridad alimentaria tienen que surgir desde las

\footnotetext{
${ }^{2}$ Los habitantes del corregimiento de Bitaco manifestaron que los cultivos de hortalizas se siembran sobre pedido.
} 
experiencias locales y regionales de autonomía para potenciarlas y afianzarlas en una construcción de abajo hacia arriba $^{3}$.

Por otro lado, en el corregimiento de Bitaco, la mayoría de los predios son fincas con destino para recreación y descanso, existe un alto componente de inmigración de personas procedentes de la ciudad de Santiago de Cali hacia el corregimiento, en especial los fines de semana, con la idea de tener un ambiente más rural y descansar de las actividades agitadas de la ciudad. La mayoría de las fincas de veraneo entre semana son habitadas por familias que operan como vigilantes o cuidanderos de las casas, siendo habitantes del corregimiento de Bitaco.

En la tabla 1 se observa que el corregimiento de Bitaco cuenta con el $93 \%{ }^{4}$ (3000 Ha) de su tierra destinada a un uso agropecuario y el 0,09\% del territorio $(2,96 \mathrm{Ha})$ en uso habitacional (Tabla 1), actualmente, la mayoría de los predios en el corregimiento de Bitaco son destinados a casas de veraneo y recreación, se observa poca presencia de fincas con actividad agrícola. (Figura 2).

\footnotetext{
${ }^{3}$ Luis Fernando Londoño. Profesor, Universidad del Cauca, Facultad de Ciencias Agropecuarias. TULL, Grupo de Investigación para el Desarrollo Rural. Popayán.

${ }^{4}$ Datos obtenidos del Geoportal del Instituto Geográfico Agustín Codazzi -IGAC-. Recuperado de

http://ssiglwps.igac.gov.co/ssigl2.0/visor/galeria.r eq? mapaId=23.
}

Tabla 1. Usos económicos de la tierra en el corregimiento de Bitaco

\begin{tabular}{|c|c|c|}
\hline Tipo de uso & $\begin{array}{l}\text { Hectárea } \\
\text { (ha) }\end{array}$ & $\begin{array}{c}\text { Porcentaje } \\
(\%)\end{array}$ \\
\hline Agropecuario & 3009,1 & 93,5 \\
\hline $\begin{array}{l}\text { Educativo-Institución } \\
\text { educativa }\end{array}$ & 9,30 & 0,28 \\
\hline Habitacional & 2,96 & 0,09 \\
\hline Institucional & 141,1 & 4,38 \\
\hline Lote no Urbanizable & 0,84 & 0,02 \\
\hline Recreacional & 36,93 & 1,14 \\
\hline Religioso & 16,6 & 0,51 \\
\hline Total & 3217 & 100 \\
\hline
\end{tabular}

Fuente: IGAC (2005) 
Fuente: (Instituto Geográfico Agustin Codazzi, 2005)

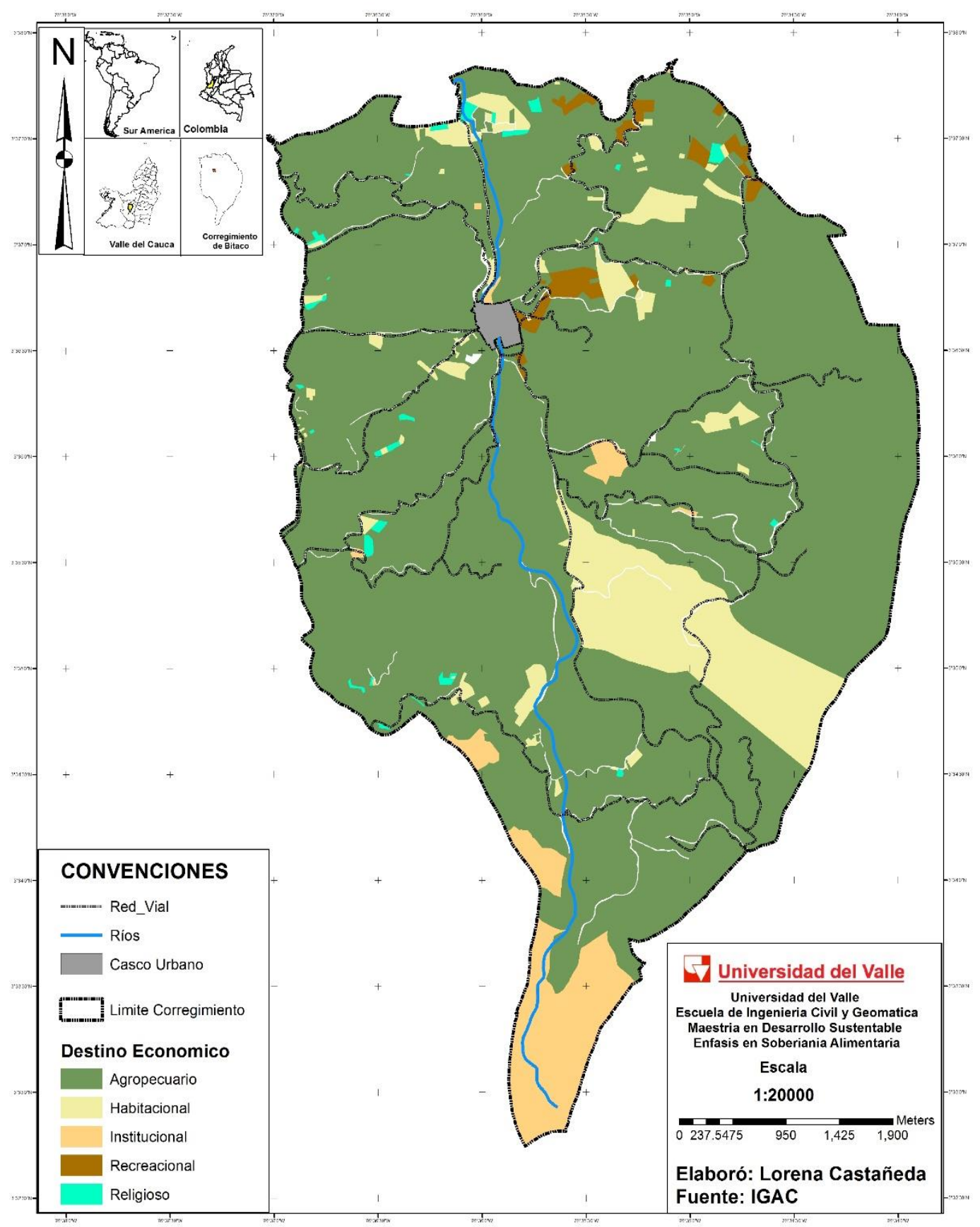

Figura 2. Destino económico del corregimiento de Bitaco 


\section{Experiencias de agricultores en el corregimiento de Bitaco}

El señor Hugo Gutiérrez, nació en el corregimiento de Bitaco, su núcleo familiar está compuesto por cuatro personas, sus ingresos económicos mensualmente son un salario mínimo, él se hace a cargo económicamente de todo su núcleo familiar. Su finca mide menos de una hectárea, no tiene gran variedad de cultivos, ya que el verano afecto la mayor parte de ellos, lo que impidió sembrar sus productos (arracacha y frijoles), estos productos son para consumo propio, no existe ningún excedente de ellos para la venta. Actualmente tiene un pequeño cultivo de café, este producto lo vende a la federación nacional de cafeteros en el municipio de la Cumbre. Los alimentos para consumo propio son comprados en la ciudad de Santiago de Cali. El tiempo que le dedica a trabajar en su finca es de una a dos horas diarias, ya que se encuentra realizando otro tipo de actividad laboral diferente a la agricultura, pues manifiesta que, si su economía se basara en la venta de café, no sería suficiente para mantener a su familia.

20 años atrás su familia se sostenía económicamente de la producción agrícola que generaba su finca; en ese entonces cultivaban maíz, frijoles, café y tenían una que otra gallina, todo esto era para consumo propio. A estos cambios significativos, el señor Hugo los atribuye a diferentes factores, entre ellos; el cambio climático, la temporada de verano afecto en gran medida la producción de alimentos y la baja del café afecto a más de un agricultor en la zona.

Otra experiencia de agricultores en el corregimiento de Bitaco es el señor Javier, el lleva 24 años en el corregimiento, su núcleo familiar se conforma por tres personas el cual, solo tiene a cargo una de ellas, su ingreso mensual es de un salario mínimo. Los alimentos para el consumo diario los compra en el municipio de la Cumbre. Actualmente tiene cultivado plátano guayabo, yuca, maíz y guineo, diariamente le dedica de una a dos horas a su finca, puesto que se dedica a otra actividad laboral diferente a la de agricultura. Todos estos alimentos son para consumo propio, ninguno de ellos es para la venta.

El señor Javier expresa que hace 20 años atrás los cultivos que más predominaban era el café junto con cultivos de plátano, en ese tiempo prevalecían las fincas cafeteras, hecho que fue desapareciendo a través del tiempo. Uno de los principales cambios que se le atribuye a la desaparición de las fincas cafeteras, fue la venta de fincas por lotes, sumándole también la falta de mano de obra para recolectar el café, esto conllevó a que los agricultores perdieran la cosecha y dejaran de cultivar el café para ser remplazado por cultivos de ciclos cortos, como los cultivos de hortalizas. No obstante, otro factor que afecto en gran medida los cambios en la producción agrícola, según testimonio por el señor Javier, es el cambio climático que ha enfrentado el país. 
Finalmente se entrevistó al señor Rodrigo Vargas, botánico de profesión y agricultor orgánico que llego al corregimiento de Bitaco hace 24 años. En sus inicios como agricultor comenzó con el cultivo de tomate, su cosecha no tuvo éxito, la calidad del tomate no era la mejor; hubo un hecho relevante que lo llevó a replantearse si continuaba con este cultivo; él, como agricultor novato, para mantener su cultivo libre de plagas y obtener un producto de buena calidad optaba por aplicar agroquímicos en gran cantidad. En labores de recolecta del cultivo de tomate, encontraba continuamente aves muertas que llegaban alimentarse en la zona. Este hecho relevante, lo llevó a replantearse y buscar un medio más orgánico y saludable para la salud humana, es por esto que llegó a la agricultura orgánica.

Rodrigo tiene alquilado 16 hectáreas por \$ 900.000 pesos mensuales. En las 16 hectáreas tiene sembrado; sábila, cúrcuma, pipilanga, moringa, frijoles, nopal y tomate cherry. Cabe aclarar, que cada uno de estos productos agrícolas son cultivados con fines medicinales. " $L a$ idea es sembrar productos que ayuden a mitigar el daño que la gente se está haciendo con lo que está comiendo, es un aporte pequeño con lo que hago, pero la idea es seguir creciendo y ayudar más a la población, no es una tarea fácil pero tampoco imposible" según testimonios del señor Rodrigo.

A la vez que el señor Rodrigo cultiva sus productos va generando un tipo de fórmula, entre estos y lo que puede ser beneficioso para la salud humana, entre ellos; problemas de azúcar, triglicéridos, ácido úrico, etc. Ninguno de estos productos agrícolas es fumigado con agroquímicos para no alterar los minerales de ellos y son cultivados de manera orgánica.

De esta manera, el señor Rodrigo expone detalladamente cuales son los beneficios de algunos de sus productos agrícolas sembrados, como, por ejemplo; la Cúrcuma, este producto cuenta con muchísimas ventajas sobre la salud humana, es un excelente antioxidante más que la vitamina $\mathrm{E}$, regenera las células, ayuda a prevenir y detener la velocidad del cáncer, baja los niveles de azúcar, los triglicéridos, ácido úrico y ayuda con problemas de artrosis. Todos estos productos son comercializados en la ciudad de Santiago de Cali, para supermercados como Mercamio y Mercatodo. No obstante, el tiempo dedicado a su finca es más de 6 horas, pues económicamente se sostiene con esta actividad agrícola.

Por otro lado, el agricultor plantea que existen cuatro alimentos que están matando a las personas, entre ellos; tomate de cocina como líder, el lulo, el tomate de árbol y la habichuela, esto es lo que más consume las personas. Son alimentos muy sensibles a recibir el toxico, debido a su piel delgada, lo que absorbe con mayor facilidad el agroquímico. Alimentos como estos, son dados frecuentemente a los niños, a medida que van creciendo, van creciendo con un sistema inmune deficiente, 
generando en el tiempo cualquier tipo de enfermedad, como cáncer, alteración en el sistema nervioso y alteración en las hormonas.

Hace varios años recibía cursos de capacitación sobre estudios de botánica y homeopatía por parte del SENA $^{5}$ actualmente no cuenta con ninguna asistencia técnica para cultivos agrícolas (Figura 3) Este agricultor, tiene la colaboración de tres personas más para actividades varias que se realizan diariamente en la finca para el cuidado y mantenimiento de los cultivos. El jornal lo paga dependiendo de la experiencia que tenga cada una de las personas; el que menos experiencia tenga paga de $\$$ 22.600 pesos hasta $\$ 24.000$ pesos, si la persona tiene más tiempo trabajando y tiene más experiencia, paga el jornal a $\$ 29.000$ pesos.

Fuente: Elaboración propia.

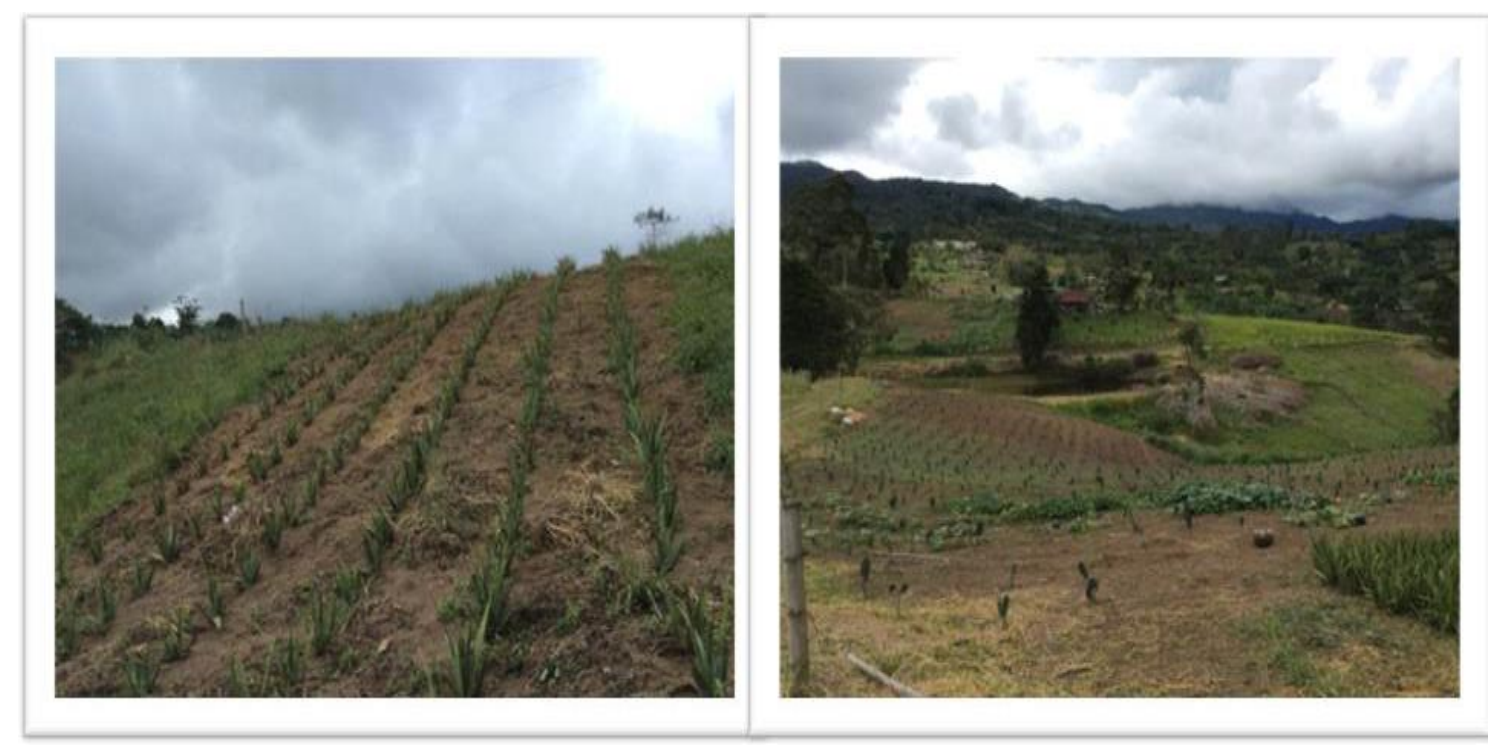

Figura 3. Cultivos de Sábila y Nopal

\footnotetext{
${ }^{5}$ SENA Servicio Nacional de Aprendizaje
} 
Pese a que el corregimiento de Bitaco cuenta con gran variedad de alimentos, que pueden suplir alimentariamente a la comunidad, no es común que los diferentes alimentos cultivados sean vendidos en la zona. La comunidad compra sus alimentos fuera del corregimiento ya sea en el municipio de la Cumbre o en la ciudad de Santiago de Cali. Es claro que existe una problemática en cuanto a la comercialización y distribución de los productos, pues la mayoría son fincas que siembran sobre pedido" es decir, se cultivan la cantidad de alimentos que ha sido encargada por los diferentes almacenes de cadena de la ciudad de Cali dejando algunos productos los habitantes del corregimiento de Bitaco, como es visto en el caso anterior con el agricultor orgánico; todos sus productos se van directo a los grandes súper mercados de cadena de la ciudad de Santiago de Cali.

Actualmente, no cuentan con ninguna entidad que patrocine este tipo de actividades que ayuden al pequeño agricultor a conservar y a mantener los recursos naturales. Quizás por esto, la gran mayoría de los agricultores caen en el error de destruir y sobreexplotar los recursos naturales, principalmente por la falta de información y apoyo técnico por entidades que apuesten al desarrollo económico sostenible del País.

En síntesis, sobre el análisis general de observación del territorio, se puede concluir que:
- La mayor parte de los predios en el corregimiento de Bitaco son con fines de recreación y descanso.

- Es evidente que la concentración de la propiedad de la tierra en el corregimiento de Bitaco presenta un aumentando a lo largo del tiempo.

- La falta de acceso a la tierra impide tener un buen desarrollo agrícola de la región.

- Toda la producción de alimentos que se desarrolla en el corregimiento de Bitaco es enviado y comercializado a la Ciudad de Santiago de Cali.

- El corregimiento de Bitaco, cuenta con buenas condiciones para la siembra de diferentes productos agrícolas, pero no hay excedentes de estos alimentos para los habitantes de la zona.

- Los habitantes del corregimiento de Bitaco compran sus alimentos fuera del caso urbano, sea en la ciudad de Cali o la Cumbre.

- Los pequeños agricultores alquilan tierras de grandes extensiones para ejercer diferentes actividades agrícolas.

- El cambio climático, también ha sido uno de los principales factores que ha afectado en gran medida las actividades agrícolas del corregimiento de Bitaco pues el incremento en la frecuencia y duración de época seca

- Existe un interés por parte de algunos agricultores en cambiar la 
manera en que se cultiva comercialmente, optando por una agricultura orgánica fuera de agroquímicos ya que, en un alto porcentaje lo que cotidianamente se consume está afectando la salud de las personas.
ESTADO DE LA AUTONOMÍA

\section{ALIMENTARIA EN EL CORREGIMIENTO DE BITACO}

La autonomía alimentaria (Figura 4) se establece a nivel de comunidades y familias productoras, en vez de contemplarlo a nivel nacional. Esto implica que los pequeños productores: campesinos, indígenas y afrodescendientes, no sean dependientes de otros actores externos para el acceso a su alimentación. Es así como la autonomía alimentaria hace referencia al "autogobierno de las comunidades". Este concepto rompe claramente con la concepción nacional-estatal de la soberanía alimentaria. Pero también enfatiza en un aspecto nuevo: en el control por parte de las comunidades de toda la cadena productiva, lo que implica que las familias productoras $\mathrm{y}$ las comunidades rurales no dependan de insumos externos (fertilizantes, plaguicidas y semillas), que controlen en buena parte las cadenas de comercialización y no dependan de empresas capitalistas externas ya sean nacionales o multinacionales (Perret y Zúñiga Vega, s.f). 
Fuente: Adaptado de (Perret y Zúñiga Vega, s.f).

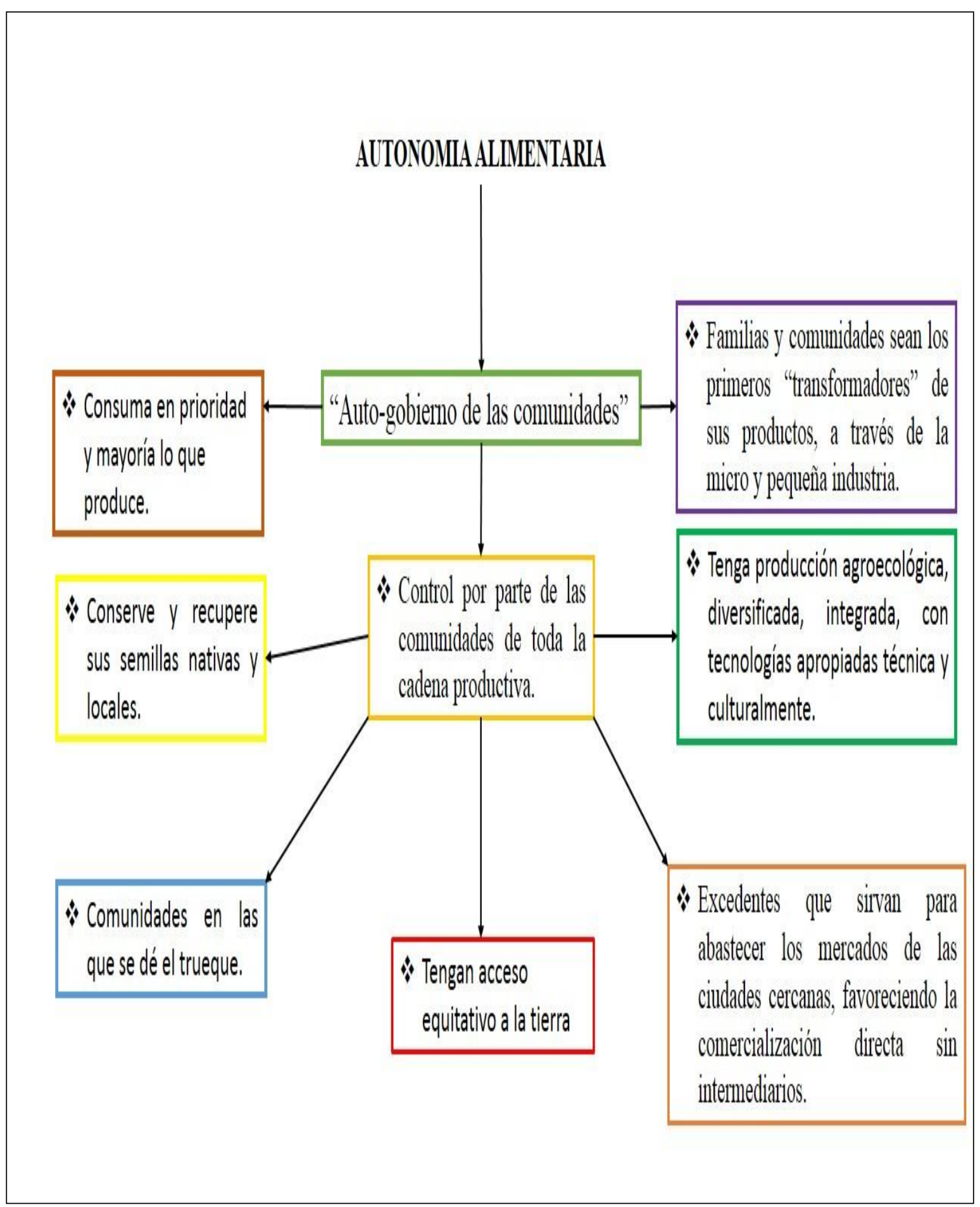

Figura 4. Estructura de la Autonomía Alimentaria. 
La autonomía alimentaria se establece a nivel de comunidades y familias productoras, en vez de contemplarlo a nivel nacional esto implica que los pequeños productores: campesinos, no sean dependientes de otros actores externos para el acceso a su alimentación. Es así como la autonomía alimentaria hace referencia al "auto-gobierno de las comunidades" este concepto rompe claramente con la concepción nacionalestatal de la soberanía alimentaria pero también, enfatiza en un aspecto nuevo: en el control por parte de las comunidades de toda la cadena productiva, lo que implica que las familias productoras y las comunidades rurales no dependan de insumos externos (fertilizantes, plaguicidas y semillas), que controlen en buena parte las cadenas de comercialización, ni de empresas capitalistas externas ya sean nacionales o multinacionales (Perret y Zúñiga Vega, s.f).

Las principales características de la autonomía alimentaria, se basan en la independencia que cada comunidad o familia productora tiene para comprar sus propios insumos, su control y conservación de las semillas, el acceso equitativo a la tierra, excedentes que sirvan para abastecer los mercados de las ciudades cercanas, lo que favorece la comercialización propia de sus alimentos, de manera directa y sin intermediarios (Figura 4).

En un ejercicio realizado por Ruiz (2015) establece que el municipio de la Cumbre tiene un índice de seguridad alimentaria medio, esta hipótesis sustentada a través de varias categorías de análisis, establece que el municipio de la Cumbre es quien más porcentaje de población vieja cuenta, al igual que mayor población rural (Figuras 5 y 6), los suelos cultivables para la Cumbre a nivel municipal presenta un nivel mínimo, lo que obstaculiza tener un buen desarrollo agrícola y económico en la región (Figura 7). Por otro lado, el índice de Gini $^{6}$ para el municipio de la Cumbre presenta un total de 0,73 el cual, a nivel municipal presenta un nivel superior lo que establece una alta concentración de la propiedad de la tierra (Figura 8).

\footnotetext{
${ }^{6}$ El índice de Gini o coeficiente de Gini es una medida económica que sirve para calcular la desigualdad de ingresos que existe entre los ciudadanos de un territorio, normalmente de un país. Se encuentra entre 0 y 1 , siendo cero la máxima igualdad y 1 la máxima desigualdad.
} 
Figura 5. Población rural

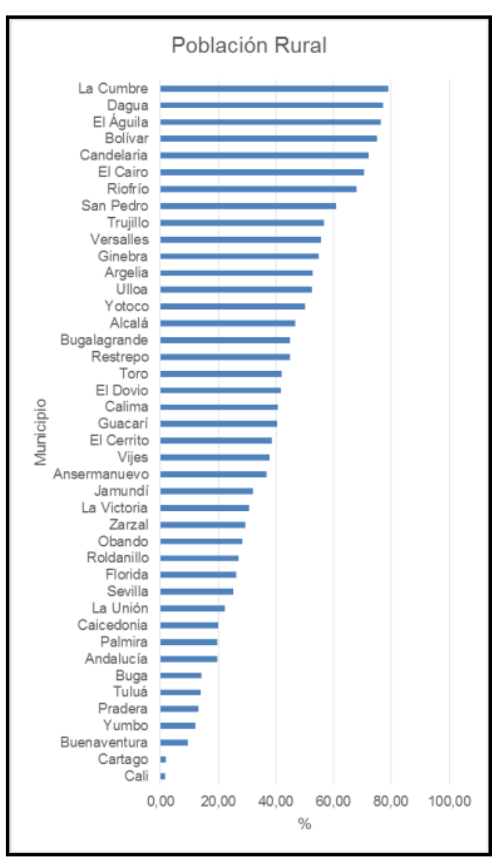

Figura 7. Población rural

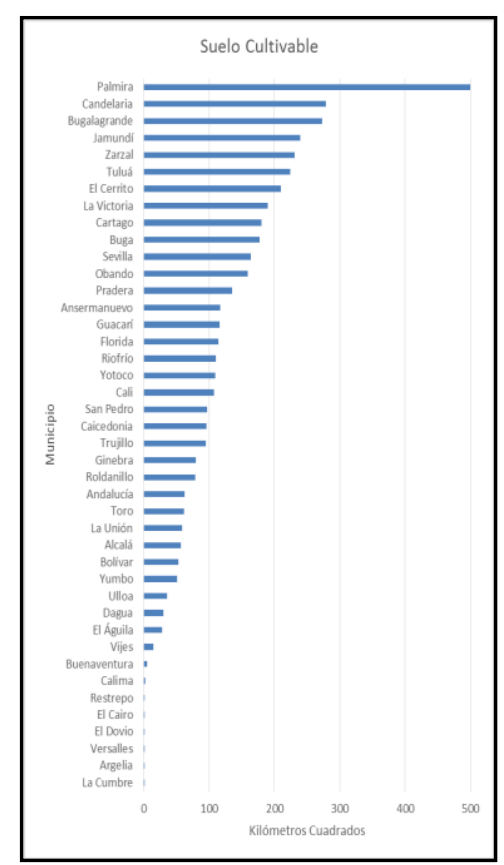

Figura 6. Índice de envejecimiento

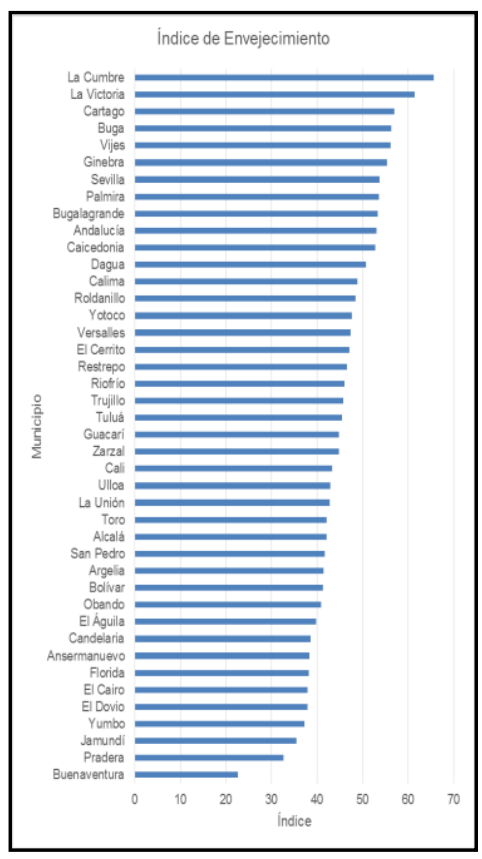

Figura 8. Gini de tierras

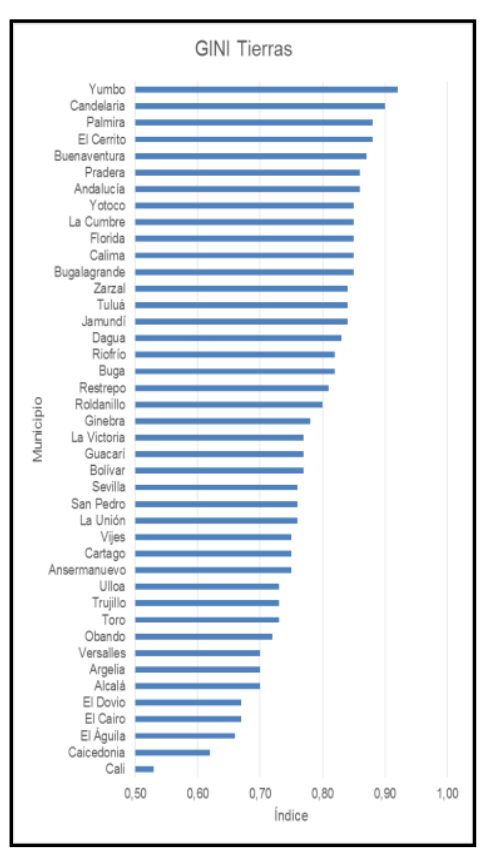

Fuente: Leonardo David Ruiz. Potencialidad de seguridad alimentaria para los municipios del Valle del Cauca 2010. 
Como se mencionó anteriormente, el municipio de la Cumbre presenta un alto índice de población mayor lo que podría afectar en un futuro el desarrollo y la producción de alimentos en el municipio, puesto que la población más joven migra del campo a la ciudad con la ilusión de encontrar nuevas oportunidades de trabajo. Para ellos, el trabajar en el campo no es visto como una buena fuente de empleo ni mejores condiciones de vida, por el contrario, piensan que la permanencia de ellos en zonas rurales es una manera de estancamiento, donde las oportunidades laborales y educativas son pocas y limitadas. Este hecho, evidencia el riesgo que tiende a desaparecer las actividades de agricultura en el campo, pues las nuevas generaciones no tienen ningún interés en continuar realizando actividades agrícolas, con los conocimientos heredados por sus padres agricultores.

De acuerdo con lo anterior, el discurso del desarrollo en las áreas rurales se ha estigmatizado como atrasadas, pero lo rural siempre ha jugado un papel importante en la configuración de la sociedad, solo que se ha visto inhibido por las políticas desarrollistas. Es el sector rural, el primer frente que tiene la sociedad para promover una relación sustentable con la naturaleza, la mayor parte de los alimentos son producidos en áreas rurales (Ruiz, 2015).

Según Ruiz (2015) si la mayoría de la población es urbana la presión de producción de alimentos recaería sobre el pequeño porcentaje de población rural, por tanto, entre mayor sea la diferencia de porcentajes de población urbana y rural menor seguridad alimentaria municipal, pues supondría la necesidad de suplir la demanda de alimentos con los recursos de otro municipio.

Por otro lado, la concentración de la propiedad ha afectado a nuestro país, hace algunos años atrás, las dinámicas actuales del capital amenazan seriamente el derecho a una alimentación adecuada. En el caso de Colombia, observamos una situación problemática en donde las comunidades indígenas, afro descendientes y campesinas están siendo despojadas de sus territorios, para ser entregados a monopolios y empresas trasnacionales, favoreciéndose de la extracción de todos los recursos naturales de la región y desfavoreciendo en este caso a las comunidades campesinas, puesto que la concentración de la tierra por parte de estas empresas multinacionales ocasiona una notoria reducción de la misma impidiendo desarrollar las diferentes actividades agrícolas productivas de los campesinos.

Para Torres, (1987) al avanzar el conocimiento sobre el problema del hambre y la desnutrición en relación con la producción mundial de alimentos, se hace evidente y más fuerte la convicción de que el problema no está en la producción de alimentos si no en los factores de distribución y acceso a los 
alimentos. Se ha hecho evidente la necesidad de integrar los problemas de la agricultura con otros de gran trascendencia para el desarrollo.

Los obstáculos principales para una eficiente $y$ equitativa distribución $y$ producción de alimentos radican más en la organización socioeconómica y política. El primer problema hace relación con los problemas de disponibilidad, a la relación entre la evolución de la producción y la demanda. El segundo se refiere a la presencia de sectores significativos de la sociedad que no logran satisfacer sus requerimientos nutricionales básicos, y se denominan de acceso (Torres, 1987). Aunque, la disponibilidad es muy importante, lo cierto es que el problema del hambre y la malnutrición en Colombia usualmente se relaciona con las dificultades que viven diariamente las familias para acceder a los alimentos. En Colombia, al igual que en la mayoría de países del mundo, no sufre problemas de escasez de alimentos, pero aun así el hambre se encuentra bastante presente en nuestra vida diaria (Grain,2005).

Según la FAO, la disponibilidad de alimentos, se refiere tanto a problemas estructurales como coyunturales. En el primer caso el énfasis recae en la persistencia o crecimiento de la brecha demanda-producción interna. En el caso coyuntural la preocupación está referida a fluctuaciones de precios vinculados al carácter de la oferta o las variaciones de los precios internacionales; aspectos logísticos de distribución física de la oferta (deficiencias de espacios físicos, problemas en transporte y en red de comunicación) (Torres, 1987).

En el acceso a los alimentos se hace una distinción similar. Los problemas coyunturales indican dificultades de acceso por fenómenos naturales, sequías, pérdida de empleo o de otro tipo que dificultan el consumo. Los estructurales apuntan a condiciones de pobreza e indigencia que se perpetúan en sectores específicos de la población. Es así, que la problemática es un conjunto complejo e interrelacionado de elementos constitutivos de la disponibilidad y acceso a los alimentos, que interactúan entre si y están inmersos en una realidad socioeconómica y política que les fija unos límites o un espacio determinado (Torres, 1987).

El problema alimentario, al estar configurado por aspectos coyunturales y estructurales, tanto en la disponibilidad como en el acceso y al estar determinados cada uno de ellos por factores diferentes, conduce a la búsqueda de políticas de distinta naturaleza para solucionarlo. Si el problema es básicamente de acceso estructural, su solución no se logra solamente con políticas alimentarias y requiere acciones que involucran todo el estilo de desarrollo.

En síntesis, el corregimiento de Bitaco hasta el momento no ha tenido autonomía alimentaria. Sus limitaciones para producir sus propios alimentos, hacer 
manejo de sus propias semillas, comercializar y consumir lo que se produce no es posible debido al difícil acceso a la tierra que enfrenta la población del corregimiento de Bitaco. Finalmente, pocos propietarios son dueños de casi la mitad del área del corregimiento, lo que limita en cierta medida a la población campesina realizar actividades agrícolas que garanticen su alimentación y el de su familia. Por otro lado, el proceso de expansión que propicia el área metropolitana de Santiago de Cali hacia el corregimiento de Bitaco, es otra de las principales causas que afecta a los agricultores del corregimiento y las actividades de agricultura. La mayor parte de la población decide realizar labores como mayordomos en diferentes casas del corregimiento que son con fines recreativos y turísticos, situación que dificultad aún más desarrollo agrícola de la zona.

\section{REFERENCIAS BIBLIOGRÁFICAS}

Absalón, M. (2009). Ensayos para la historia de la politica de tierras en Colombia, de la Colonia a la creacion del frente Nacional. Bogota: Universidad Nacional de Colombia.

Alcaldía de la Cumbre Valle del Cauca. (26 de mayo de 2014). Recuperado de de http://www.lacumbre- valle.gov.co/informacion_general. shtml\#economia.

Beltrán Gutiérrez, Y. K., y Gutiérrez

Rozo, D. (2007). Cambios en la estructura productiva agrícola en las regiones Caribe, CentroOriente de Colombia en el periodo 1980 - 2004. Bogotá: Universidad de la Salle.

Corporación Autónoma Regional del Valle del Cauca. CVC. (2003). Sistema de Información geografica de la unidad de manejo de la cuenca alto -Dagua-La Cumbre- Restrepo. Cali.

Corporación Autónoma Regional del Valle del Cauca. CVC. (2006). Plan de manejo participativo Reserva Forestal de Bitaco. Santiago de Cali.

Corporacion Autónoma regional del Valle del Cauca. CVC. (Octubre de 2017). Visor geográfico avanzado. Obtenido de https://www.geo.cvc.gov.co/visor _avanzado/

Díaz Bravo, L., Torruco García, U., Martínez Hernández, M., \& Varela Ruiz, M. (2013). La entrevista, recurso flexible y dinámico. Elsevier.

Enríquez, O. O. (2004). Historia del Municipio de la Cumbre. Cali: Anzuelo Ético Ediciones.

Franco, A., y De los Ríos, I. (2011).

Reforma agraria en Colombia: evolución 
histórica del concepto. Hacia un enfoque integral actual. Cuad. Desarrollo Rural, 8(67): 93-119.

Grain. (2005). Soberania alimentaria y sistema alimentario mundial. Revista Biodiversidad, (47), 1-4. Recuperado de http://www.grain.org/es/article/ent ries/1086-soberania-alimentaria-ysistema-alimentario-mundial.

Idárraga Bahos, A. D. (2012). Buenas practicas agricolas desde un enfoque agroecologico como aporte al desarrollo local sustentable de la organización de asocampoalegre en el corregimiento de campoalegre, Andalucia, Valle del Cauca (trabajo de grado). Universidad Autónoma de Occidente, Santiago de Cali, Colombia.

Instituto Geográfico Agustin Codazzi. (Octubre de 2005). Datos abiertos agrologia. Obtenido de https://geoportal.igac.gov.co/conte nido/datos-abiertos-agrologia

López, L. M. (22 de marzo de 2004). La cartografia social y su aplicación a la planificación Municipal Regional. Recuperado el 30 de octubre de 2015.

Machado, A. (1998). La cuestion agraria en Colombia a finales del milenio. Bogota: Ancora.

Perret, C. y Zúñiga Vega, Z. (s.f). ¿SEGURIDAD, SOBERANÍA O
AUTONOMÍA ALIMENTARIA?

Recuperado de https://cyrilperret.files.wordpress. com/2011/12/artc3adculoautonomc3ada-alimentariaversic3b3n-final.pdf.

Programa de las Naciones Unidas para el desarrollo. PNUD. (2012). La hora de la Colombia rural. Hechos de paz. Recuperado de https://reliefweb.int/sites/re liefweb.int/files/resources/ Full_Report_3049.pdf.

Ruiz, L. D. (2015). Potencialidad de seguridad alimentaria para los municipios del Valle del Cauca, Santiago de Cali. Cali, Colombia: Universidad del Valle.

Torres, A. M. (1987). El sistema agroalimentario una vision integral de la cuestion agraria en america latina. Bogota, Colombia: Presencia. 